\title{
GW23-e2681 THE EFFECTS OF TELMISARTAN ON SERUM LEVEL OF COPEPTIN IN PATIENTS WITH CHRONIC HEART FAILURE
}

doi:10.1136/heartjnl-2012-302920v.10

Zhong Jianghua, Lu Shijuan, Chen Moshui, Du Zhijun, Wu Miao, Sun Dingjun, Zhong Jianghua. Department of Cardiology of Haikou People' Hospital, Affiliated Haikou Hospital of Xiangya Medical School of Central South University

Objectives To study the effects of telmisartan on serum level of copeptin in patients with chronic heart failure (CHF).

Methods 72 patients with CHF were divided into CHF control group $(n=40)$ and CHF treatment group $(n=32)$, and another 30 healthy adults were selected as normal control group. Patients in the CHF control group were given conventional medicine treatment such as digoxin, diuretics, nitrates and so on. Patients in the CHF treatment group were given orally telmisartan $(80 \mathrm{mg} /$ day) for 6 months besides conventional medicine treatment. Left ventricular end-diastolic diameter (LVEDD), left ventricular ejection fraction (LVEF), the level of high sensitivity C-reactive protein (hsCRP) and copeptin were evaluated in all subjects before and after treatment.

\section{Results}

1. Compared to those in the normal control group, LVEDD, LVEF, hs-CRP and copeptin were significantly different $(p<0.05)$ in the both CHF control group and CHF treatment group before treatment, and LVEF is negative related to level of copeptin $(R=-0.38, p<0.05)$.

2. Although there was no change for LVEDD $(p>0.05)$ after treatment for 6 months in the both CHF control group and CHF treatment group, LVEF were improved $(p<0.05)$ and hs-CRP and copeptin were significantly decreased $(p<0.05)$.

3. After treatment for 6 months, LVEF was higher and hs-CRP and copeptin were significantly lower $(p<0.05)$ in the CHF treatment group compared to those in the CHF control group.

Conclusions Telmisartan can improve heart function of patients with CHF by decreasing serum level of copeptin and alleviating inflammatory reaction. 